\title{
Nonlinear doublon production in a Mott insulator — Landau-Dykhne method applied to an integrable model
}

\author{
Takashi Oka \\ Department of Physics, University of Tokyo, Hongo, Tokyo 113-0033, Japan and \\ Department of Physics, Harvard University, Cambridge, MA 02138, USA
}

(Dated: August 27, 2018)

\begin{abstract}
Doublon-hole pair production which takes place during dielectric breakdown in a Mott insulator subject to a strong laser or a static electric field is studied in the one-dimensional Hubbard model. Two nonlinear effects cause the excitation, i.e., multi-photon absorption and quantum tunneling. Keldysh crossover between the two mechanisms occurs as the field strength and photon energy is changed. The calculation is done analytically by the Landau-Dykhne method in combination with the Bethe ansatz solution and the results are compared with those of the time dependent density matrix renormalization group. Using this method, we calculate distribution function of the generated doublon-hole pairs and show that it drastically changes as we cross the Keldysh crossover line. After calculating the tunneling threshold for several representative one-dimensional Mott insulators, possible experimental tests of the theory is proposed such as angle resolved photoemission spectroscopy of the upper Hubbard band in the quantum tunneling regime. We also discuss the relation of the present theory with a many-body extension of electron-positron pair production in nonlinear quantum electrodynamics known as the Schwinger mechanism.
\end{abstract}

PACS numbers: 78.47.J-,02.30.Ik,71.27.+a,03.75.Kk

\section{INTRODUCTION}

"Nonequilibrium strongly correlated systems" is becoming an important field of study in condensed matter physics [1 12]. These systems offer a testbed for theoretical advances such as the extension of the linear response paradigm to nonlinear processes [13 26]. We can experimentally induce a nonequilibrium state in photoinduced phase transitions in solids [1, 2] as well as in the dynamics of cold atoms 9 12. The photo-induced insulator to metal transition in Mott insulators has generated substantial interest because it is one of the most basic nonequilibrium phenomena in strongly correlated systems [1, 2] . The response of Mott insulators subject to strong external fields has been studied experimentally. Initially, the motion of particles (electrons or atoms) is frozen by strong repulsion, and the ground state is a Mott insulator 27]. Perturbations (electric field or lattice modulation) excite pairs of doublons (= doubly occupied site) and holes (= sites with no electrons; we do not call this state "holon" because this is a Bethe ansatz terminology that is used later), and when their density becomes sufficiently high, "melting" of the Mott state occurs leading to an insulator-to-metal transition [1]. Quite recently, the insulator-to-metal transition was realized by a terahertz laser in vanadium dioxide, which is a candidate material for a Mott transition [8]. Since the photon energy is far below the optical gap, the excitation mechanism is expected to be a nonlinear process. These experiments give us strong motivation to develope a theory for nonlinear excitations in strongly correlated systems.

The purpose of this study is to gain an analytical understanding of the excitation process when a strong electric field is applied to a Mott insulator. The effects of strong electric fields on Mott insulators have been studied extensively in theory via the fermionic Hubbard model using numerical methods such as exact diagonalization [13, 21], the time-dependent density matrix renormalization group (td-DMRG) 14, 15, 20], and nonequilibrium dynamical mean field theory [17, 18]. These studies reveal the following consensus. Doublon-hole pairs (dh-pairs) are created by strong electric fields, and for DC-electric fields, production rate (or ground state decay rate) shows a threshold behavior 13, 14. This behavior seems to be universal and independent of dimensions, e.g., Refs. [13 15, 20] (one-dimensional (1-D) studies) and Ref. [17] (infinite-dimensional studies). If we denote tunneling threshold by $F_{\text {th }}$, for small electron repulsion $U$, it behaves as $F_{\text {th }} \propto \Delta_{\text {Mott }}^{2}$, where $\Delta_{\text {Mott }}$ is the Mott gap. We can obtain this expression by applying the Landau-Zener formula to many-body energy levels 13]. For AC-electric fields, it was mentioned in Ref. 21] that there is a crossover from a weakly excited state to a strongly excited state with increasing field strength. Another interesting observation was made regarding the bosonic Hubbard model with lattice modulation, where the authors calculated energy absorption rate using td-DMRG [12]. The absorption peaked around $\Omega \sim N U$ ( $\Omega$ : modulation frequency, $N$ : integer) broadened as modulation intensity increased. The broadening is clearly a nonlinear effect.

In this study, we examine the 1D Hubbard model at half-filling and the instability of the ground state in strong electric fields. The method we use is a combination of the Landau-Dykhne quantum tunneling theory [28 30] and the Bethe ansatz. This method was developed in Ref. [15] and was used to derive an analytic expression for tunneling threshold. Although not commonly employed in condensed matter, the LandauDykhne method has a long application history in areas 
of physics where quantum systems are driven out of their initial state by strong external fields. The name "strong field physics" is often used to describe this problem area in physics. We can find examples of driven systems in quantum chemistry [30], atom ionization [31, 32], quantum chaos [33], and high energy [34 41]. "Nonlinear excitations in Mott insulators" is a typical problem in "strong field physics in condensed matter" (for a review, see [42, 43]). Ideas and techniques developed in other fields prove quite useful as well.

Our problem has many common features with the electron-positron pair production problem in nonlinear quantum electrodynamics (QED) (for a review, see Ref. [41]). The concept of vacuum in high energy physics is directly translated into an "insulator" in condensed matter. Shortly after the report of the Dirac sea vacuum description, Heisenberg-Euler proposed that nonlinear response of the vacuum is described by an effective Lagrangian [34]. They also found vacuum instability against electron-positron pair production when field strength is comparable to tunneling threshold. This threshold is now called the Schwinger limit 35]. The calculation of production rate was extended from DC to AC electric fields 36 38] following an early study by Keldysh on atom ionization [31]. In particular, Popov used the Landau-Dykhne method to calculate production rate [36, 37] (for a review of this approach, see Ref. [39]). Following these "strong field physics"-studies, a universal picture emerged that, in fact, had already been noticed by Keldysh [31]. That is, there are two leading excitation mechanisms in a zero-temperature-gapped system driven by an AC-field. One is quantum tunneling, which is dominant in the DC limit, and shows a threshold behavior. This threshold is nothing but the Schwinger limit. The other mechanism is multi-photon absorption that is dominant when photon energy is relatively large. Moreover, production rate shows a power law behavior. There is a crossover between the two regimes, which is called the Keldysh crossover.

In this study, we show that the nonlinear dh-excitation in a Mott insulator also lies within the Keldysh paradigm. Using the Landau-Dykhne method combined with the Bethe ansatz, we derive an expression for the momentumresolved production rate of dh-pairs (Eq. (24) below) and calculate the total production rate $\Gamma$. In Fig. 1 we schematically plot the production rate behavior in strong AC-electric fields

$$
F(t)=F_{0} \sin \Omega t .
$$

Here, $F_{0}=e a E(>0)$ is the field strength, $e$ is the electron charge, $a$ is the lattice constant, and $\Omega$ is the photon energy. When photon energy is above the Mott gap and is resonant with the absorption spectrum, i.e., $\Omega \sim U$, we obtain the standard linear response result, i.e., $\Gamma \propto F_{0}^{2}$. In the case where the field is off-resonant $\Omega<\Delta_{\text {Mott }}$, nonlinear processes lead to dh-production. Similar to the other above-mentioned "strong field physics" examples, the two leading mechanisms are multi-photon absorption

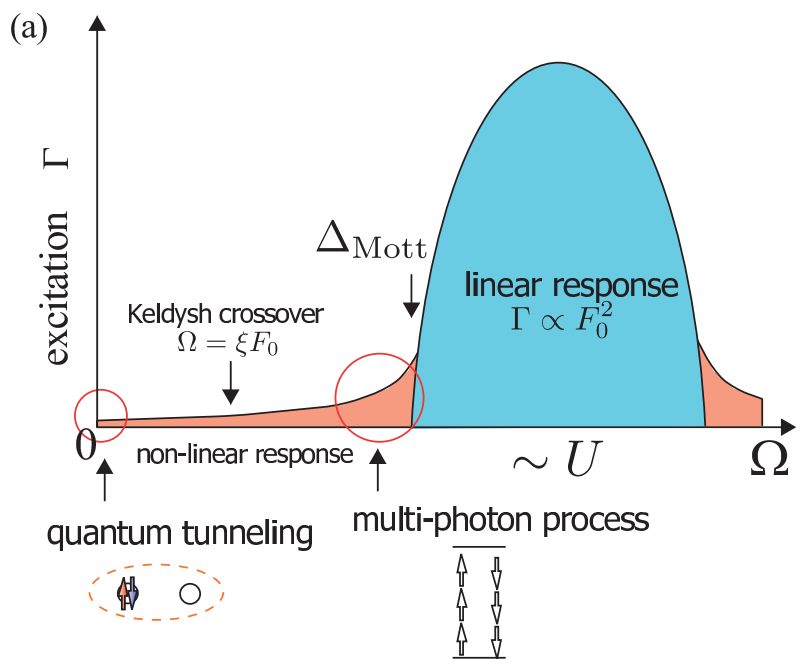

(b)

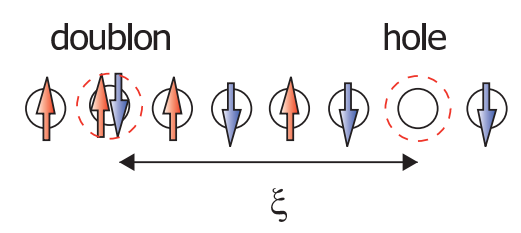

FIG. 1: (color online) (a) Schematic plot of the nonlinear optical absorption spectrum of a Mott insulator in a strong AC-electric field. $F_{0}(>0)$ is the field strength and $\Omega$ is the photon energy of the applied laser. In addition to the contribution from linear response theory (Kubo formula), sub-gap excitations occur owing to nonlinear processes. Mechanisms are quantum tunneling and multi-photon absorption. They are governed by the Mott gap $\Delta_{\text {Mott }}$ and correlation length $\xi$. (b) Correlation length $\xi$ is the typical size of doublon-hole pairs in the Mott insulating ground state. Pair production and annihilation occur during the virtual process.

and quantum tunneling. The former occurs when $\Omega$ is relatively close to the gap. Production rate has the following power law dependence on field strength

$$
\Gamma \propto\left(\frac{F_{0} \xi}{2 \pi \Omega}\right)^{2 \frac{\Delta_{\mathrm{Mott}}}{\Omega}} .
$$

Here, the power $2 \frac{\Delta_{\text {Mott }}}{\Omega}$ is twice the number of absorbed photons and the factor $\xi$ is the doublon-hole correlation length 45. In the ground state of a Mott insulator, doublon-hole pairs are created during a quantum mechanical virtual process [Fig. 1(b)]. Correlation length gives the typical size of such doublon hole pairs. When the DC limit is approached with a small $\Omega$, the leading mechanism becomes quantum tunneling. This leads to a dielectric breakdown with a threshold behavior [1315, 17]. The total production rate in this regime has the approximate form

$$
\Gamma \propto \exp \left(-\pi \frac{F_{\mathrm{th}}}{F_{0}}\right)
$$


where DC threshold (=Schwinger limit) is given by

$$
F_{\text {th }} \sim \frac{\Delta_{\text {Mott }}}{2 \xi} .
$$

We notice that the correlation length $\xi$ again plays an important role.

The paper is organized as follows. In section II after a brief introduction of the Bethe ansatz solution of the Hubbard model, we explain how to combine its information with the Landau-Dykhne method. Application to nonlinear transport in DC fields and photo-induced phase transitions in AC fields is discussed in sections III and IV] respectively. In section $\mathrm{V}$, we discuss experimental feasibility.

\section{LANDAU-DYKHNE + BETHE ANSATZ METHOD}

In this section, we extend the Landau-Dykhne + Bethe ansatz method, developed in Ref. 15], to electric fields with various laser types. The model we study is the halffilled 1D fermionic Hubbard model subject to an electric field. The Hamiltonian is given by

$$
\begin{aligned}
H(\Phi)= & -\tau \sum_{j, \sigma}\left(e^{i \Phi} c_{j+1 \sigma}^{\dagger} c_{j \sigma}+e^{-i \Phi} c_{j \sigma}^{\dagger} c_{j+1 \sigma}\right) \\
& +U \sum_{j} n_{j \uparrow} n_{j \downarrow} .
\end{aligned}
$$

The time-dependent Peierls phase $\Phi$ is related to the applied electric field by $F(t)=e a E(t)=-d \Phi(t) / d t$. We set the energy unit as the hopping amplitude, i.e., $\tau=1$. We start from the Mott insulating ground state at $t=0$ and apply the electric field for $t>0$.

The static Hubbard model can be solved exactly using the Bethe ansatz and the ground state wave function as well as excitations is well understood [44]. There are two types of elementary excitations from the halffilled ground state: (1) Gapped spinless excitations with charge $\mp e$ called antiholons and holons (2) Gapless charge neutral excitations carrying spin $\pm \frac{1}{2}$ called spinons. Physical excitations are built from these elementary excitations. In the remainder of this article, instead of using the Bethe ansatz terminology antiholon and holon, we use the more familiar names doublon and hole.

Among the excitations, we concentrate on excited states with a single doublon-hole pair, i.e., antiholonholon pairs. The states are parameterized by rapidity $k_{1}$ $\left(k_{2}\right)$ for a hole (doublon). The total energy and central momentum of these excitations are given by

$$
\begin{aligned}
\Delta E \equiv E_{d h}-E_{0} & =\varepsilon_{h}\left(k_{1}\right)+\varepsilon_{d}\left(k_{2}\right), \\
P_{\text {central }} & =p_{h}\left(k_{1}\right)+p_{d}\left(k_{2}\right),
\end{aligned}
$$

where $\varepsilon_{h, d}$ and $p_{h, d}$ are the energy and momentum of the hole and doublon, respectively. Holon energy is given by 44$]$

$$
\begin{aligned}
\varepsilon_{h}(k)= & \varepsilon_{d}(k)=U / 2+2 \cos k \\
& +2 \int_{0}^{\infty} \frac{d \omega}{\omega} \frac{J_{1}(\omega) \cos (\omega \sin k) e^{-U \omega / 4}}{\cosh (\omega U / 4)}
\end{aligned}
$$

and holon momentum is given by

$$
\begin{aligned}
p_{h}(k)= & p_{d}(k)+\pi=\frac{\pi}{2}-k \\
& -2 \int_{0}^{\infty} \frac{d \omega}{\omega} \frac{J_{0}(\omega) \sin (\omega \sin (k))}{1+\exp (U \omega / 2)} .
\end{aligned}
$$

Note that we shifted holon momentum by $\frac{\pi}{2}$, i.e., $p \equiv$ $p_{h}+\frac{\pi}{2}\left(=-p_{d}-\frac{\pi}{2}\right)$. Only states with $P_{\text {central }}=0$ can be excited by an external electric field because the momentum of the laser can be ignored. We denote a single doublon-hole pair with the hole momentum $p$ by $|p\rangle_{d h}$. In Fig. 22(a), we plot the excitation energy $\Delta E(p)$ as a function of $p$. It has a minimum at $p=0$ with a gap $\Delta_{\text {Mott }}$, i.e., the Mott gap.

An important concept in the Mott insulating phase is the correlation length $\xi$ studied by Stafford and Millis in Ref. [45] using the Bethe ansatz. In Mott insulators, each lattice site is occupied by a single electron. However, quantum fluctuations enable doublons and holes to pair create and wander around as a virtual process before they pair annihilate. This process is responsible for antiferromagnetic super exchange coupling. Correlation length is intuitively the size of the doublon-hole pair in the ground state wave function [Fig. 1(b)]. If we consider a finite system of size $L$, charge stiffness (Drude weight) decays as

$$
D_{c}(L) \propto \exp (-L / \xi)
$$

In other words, if the system is small enough compared to $\xi$, it behaves as a metal because the carriers (doublons and holes) can transport current. Another important property is that Green's function $G\left(\left|x-x^{\prime}\right|\right)=$ $\left\langle 0\left|c_{x^{\prime} \sigma}^{\dagger} c_{x \sigma}+c_{x \sigma}^{\dagger} c_{x^{\prime} \sigma}\right| 0\right\rangle$ decays as follows [45]:

$$
G(|x|) \sim \exp (-|x| / \xi) .
$$

The exact expression for $\xi$ is given by 45 ]

$$
1 / \xi=\frac{4}{U} \int_{1}^{\infty} d y \frac{\ln \left(y+\sqrt{y^{2}-1}\right)}{\cosh (2 \pi y / U)} .
$$

In Fig. 3(a), we plot $\xi$ as well as $\Delta_{\text {Mott }}$ as a function of $U$. In the small $U$ limit, it behaves as

$$
\lim _{U \rightarrow 0} \xi=\frac{2 t+U / 2 \pi+\cdots}{\Delta(U, t)}
$$

whereas the strong-coupling limit is given by

$$
\xi^{-1}=\ln (U / a t) \quad U \rightarrow \infty
$$

with $a=[\Gamma(1 / 4) / \sqrt{2 \pi}]^{4} \simeq 4.377$. 

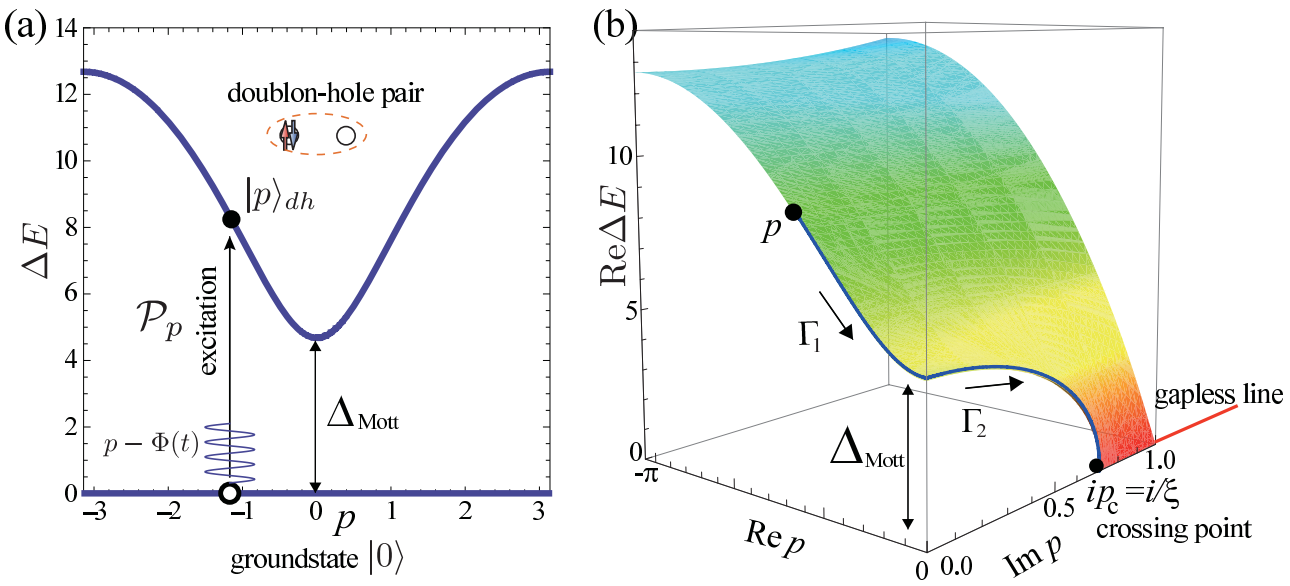

FIG. 2: (color online) (a) The excitation energy $\Delta E$ of the doublon-hole pair as a function of the hole momentum $p$ in the $U=8$ Hubbard model. $\mathcal{P}_{p}$ is the tunneling probability to create a state $|p\rangle_{d h}$ from $|0\rangle$. (b) The real part of the excitation energy plotted for complex $p$. The $\operatorname{Im} p=0$ slice is equivalent to the left half of (a). The gap closes at the level crossing point $p=i p_{\mathrm{c}}$ where a gapless line starts. Paths $\Gamma_{1}$ and $\Gamma_{2}$ are used in the integral in Eq. (25).
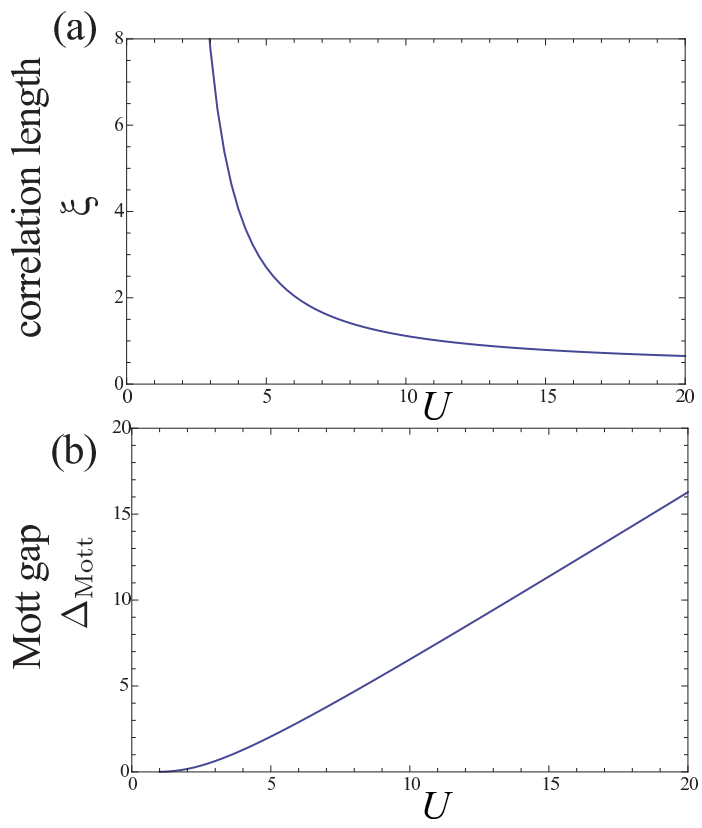

FIG. 3: (color online) (a) Correlation length $\xi$ [45] and (b) Mott gap $\Delta_{\text {Mott }}$ (Lieb-Wu solution) of the 1D Hubbard model at half-filling.

Next, we consider time evolution. We start from the ground state $|0\rangle$ and apply an electric field described by a time-dependent phase via $F(t)=e a E(t)=-d \Phi(t) / d t$. After a tunneling process, the wave function takes the form

$$
|\Psi\rangle \sim e^{-i \alpha} \sqrt{\mathcal{N}}|0\rangle+\sum_{p} \sqrt{\mathcal{P}_{p}} e^{-i \beta_{p}}|p\rangle_{d h}+\ldots,
$$

$\left(\alpha, \beta_{p}\right.$ are phases) where the ground state amplitude decreases as $\sqrt{\mathcal{N}} \sim \prod_{p} \sqrt{1-\mathcal{P}_{p}}[14]$. $\mathcal{P}_{p}$ is the momentum resolved tunneling probability of the doublon-hole pair with momentum $p$ [Fig. 2 (a)]. The omitted term “..." contains excitations to states with multiple doublon-hole pairs as well as spin excitations.

One can calculate the tunneling probability $\mathcal{P}_{p}$ in the Hubbard model by the Landau-Dykhne method [15]. The Landau-Dykhne method [28, 30] (for a textbook and useful reference see Ref. [32] and [46], respectively) has been derived from the adiabatic perturbation theory. We denote the adiabatic eigenstates of $H(\Phi)$ by $|0 ; \Phi\rangle$ and $|p ; \Phi\rangle_{d h}$ where

$$
\begin{aligned}
H(\Phi)|0 ; \Phi\rangle & =E_{0}(\Phi)|0 ; \Phi\rangle \\
H(\Phi)|p ; \Phi\rangle_{d h} & =E_{d h}(p ; \Phi)|p ; \Phi\rangle_{d h}
\end{aligned}
$$

is satisfied. Because $p$ is a good quantum number, states with different $p$ are orthogonal to each other. Thus, by ignoring multiple pair states, we can study each excitation channel independently. This means that we can study the tunneling process in a Hilbert space spanned by two states, i.e., the problem reduces to solving the time-dependent Schrödinger equation with a solution of the form

$$
|\Psi(t)\rangle=a(t)|0 ; \Phi(t)\rangle+b(t)|p ; \Phi(t)\rangle_{d h}
$$

(initial condition $a(0)=1, b(0)=0$ ). This significantly simplifies the problem. Landau-Dykhne's tunneling theory states that the tunneling probability $\left(\left|b\left(t_{\text {end }}\right)\right|^{2}\right)$ between two quantum levels is given by

$$
\mathcal{P}_{p}=\exp \left(-2 \operatorname{Im} \mathcal{D}_{p}\right)
$$

where

$$
\mathcal{D}_{p}=\int_{\gamma}\left[E_{d h}(p ; \Phi(t))-E_{0}(\Phi(t))\right] d t
$$

is the difference between the dynamical phase of the ground state and the excited state. In our problem, 
this expression can be simplified because the effect of the Peierls phase on the adiabatic solutions is expressed simply by replacing the momentum $p$ by $p-\Phi$. Thus, we have

$$
|p ; \Phi\rangle_{d h}=|p-\Phi\rangle_{d h}, E_{d h}(p ; \Phi)=E_{d h}(p-\Phi),
$$

which leads to $\mathcal{D}_{p}=\int_{\gamma} \Delta E(p-\Phi(t)) d t$, where $\Delta E$ is defined in Eq. (6).

An interesting point of the Landau-Dykhne formula [Eq. (19)] is that tunneling probability depends on the imaginary part of the dynamical phase difference. Integration path $\gamma$ starts from $t=0$ and ends at a critical time $t=t_{c}$, where level crossing takes place. Because we are dealing with a gapped system, level crossing does not occur for real $t$; instead, it occurs at a complex time when

$$
\Delta E\left(p-\Phi\left(t_{c}\right)\right)=0
$$

is satisfied. When $t$ is a complex number, the corresponding Peierls phase is also complex. The Hubbard Hamiltonian [Eq. (5D)] with a complex Peierls phase is a nonHermitian lattice model where the absolute values of the left and right hoppings are unequal. The ground state wave function of the non-Hermitian model was studied by Fukui and Kawakami in ref. 47]. In Fig. 2(b), we plot $\operatorname{Re} \Delta E$ for complex $p$. The level crossing is found at a point $i p_{c} \equiv p-\Phi\left(t_{c}\right)$. The momentum of the level crossing point is related to correlation length by [45, 48]

$$
p_{c}=1 / \xi,
$$

i.e., they are the inverse of each other. In a noninteracting system, this is a very natural relationship, which states that localization length is the inverse of complex momentum and a wave function decays as $e^{i k x}=e^{-\kappa x}$ when $k=i \kappa$. However, the surprise here is that this concept can be extended to a many-body system in a straightforward manner.

The expression for tunneling probability becomes physically clearer when we change variables in the integral from time $t$ to the Peierls phase $\Phi$. Using the Jacobian $\frac{d t}{d \Phi}=-1 / F$, where $F$ is the electric field expressed as a function of $\Phi$, we are led to the expression

$$
\mathcal{P}_{p}=\exp \left(-2 \operatorname{Im} \int_{0}^{\Phi_{c}} \Delta E(p-\Phi) \frac{-1}{F(\Phi)} d \Phi\right),
$$

which is the main result of this study. We keep the minus sign in Eq. (24) as a reminder that the factor in the exponential is negative. This expression is a direct descendant of V. S. Popov's original expression for the tunneling probability in the massive Dirac model [37]. The difference here is that the one-body energy level is replaced by the many-body level obtained by the Bethe ansatz. One can use Eq. (24) to study excitations not only for DC-fields but also for various other fields. This

\begin{tabular}{|c|c|c|}
\hline type & $F(\Phi)$ & attempt frequency $f$ \\
\hline DC-field & $F_{0}$ & $F_{0} / 2 \pi$ \\
\hline AC-field & $\pm \sqrt{F_{0}^{2}-\Omega^{2} \Phi^{2}}$ & $\Omega / 2 \pi$ \\
\hline single pulse & $F_{0} \cosh ^{-2}(t / \sigma) F_{0}\left(1-\frac{\Phi^{2}}{\sigma^{2} F_{0}^{t}}\right)$ & 1(single process) \\
\hline
\end{tabular}
can be achieved by simply replacing the function $F(\Phi)$
TABLE I: Models of electric fields. $F_{0}$ is the field strength, $\Omega$ the photon energy and $\sigma$ the pulse duration.

in the formula. Table $\square$ shows the models of the electric fields we use in this study.

There is an arbitrariness in the $\Phi$ integral in Eq. (24). Because $\mathcal{D}_{p}$ is a complex integral of an analytic function, one can deform its path as long as the end points are fixed and no singular point is crossed. The most natural path that simplifies the calculation is the one that goes from $p$ to $0\left(\Gamma_{1}\right)$ and then from 0 to $i p_{\mathrm{c}}\left(\Gamma_{2}\right)$ as shown in Fig. 2(b). On this path, $\Delta E$ is always real. The integral is divided into two

$$
\mathcal{D}_{p}=\int_{0}^{\Phi_{c}} \Delta E(p-\Phi) \frac{-1}{F(\Phi)} d \Phi=\mathcal{D}_{p 1}+\mathcal{D}_{p 2},
$$

corresponding to $\Gamma_{1}$ and $\Gamma_{2}$, and the two contributions can be written as real integrals

$$
\begin{gathered}
\operatorname{Im} \mathcal{D}_{p 1}=\int_{p}^{0} \Delta E(l) \operatorname{Im}\left(\frac{1}{F(p-l)}\right) d l \\
\operatorname{Im} \mathcal{D}_{p 2}=\int_{0}^{p_{c}} \Delta E(i l) \operatorname{Im}\left(\frac{1}{F(p-i l)}\right) d l .
\end{gathered}
$$

In these integrals, the variable $l$ is real. We can numerically perform the integration, and in some simple cases, we can derive analytical expressions, as we will see later.

We now comment on the relationship between tunneling probability and production rate. The tunneling probability $\mathcal{P}_{p}$ is defined for a single excitation attempt. To promote it to a "rate", we must multiply it with the attempt frequency $f$, i.e., the number of events per unit time. Then, momentum resolved production rate is given by

$$
\Gamma_{p}=f \mathcal{P}_{p}
$$

In the case of DC fields, physical momentum, defined on the interval $(-\pi, \pi]$, evolves as $p-\Phi(t)=p+F_{0} t$. Thus, the time period between tunneling events is $2 \pi / F_{0}$, which is nothing but the period of the Bloch oscillation. In this case, the attempt frequency is given by its inverse $f=F_{0} / 2 \pi$. As for the AC field with photon energy $\Omega$, the attempt frequency is $f=\Omega / 2 \pi$. When we consider a single pulse, we simply set $f=1$. Table $\llbracket$ summarizes attempt frequency and the Jacobian for several models of electric fields.

The total production rate is defined by

$$
\Gamma=f \int_{-\pi}^{\pi} \frac{d p}{2 \pi} \mathcal{P}_{p} .
$$

The total production rate is an important quantity because it is comparable to quantities obtained from with 
other methods such as td-DMRG. First, the total production rate gives the lowest order approximation for the ground state decay rate [14]. The ground-stateto-ground-state transition amplitude (fidelity amplitude) for a time-dependent Hamiltonian is defined as

$$
\begin{aligned}
\Xi(t)= & \left\langle 0 ; \Phi(\tau)\left|\hat{T} e^{-\frac{i}{\hbar} \int_{0}^{t} H(\Phi(s)) d s}\right| 0 ; \Phi(0)\right\rangle \\
& \times e^{\frac{i}{\hbar} \int_{0}^{\tau} E_{0}(\Phi(s)) d s},
\end{aligned}
$$

where $\hat{T}$ stands for time ordering. When the ground state is unstable in the external driving force, the absolute value of the amplitude decays exponentially as $(D=1$ 1D)

$$
|\Xi(t)| \sim e^{-t L^{D} \Gamma_{\text {g.s.decay }} .}
$$

The total production rate defined in Eq. (31) agrees with the ground state decay rate up to the higher order tunneling process, i.e., $\Gamma_{\text {g.s.decay }}=\Gamma+\ldots$, where terms such as $\left(\mathcal{P}_{p}\right)^{2}$ are neglected. For example, the ground state decay rate of a band insulator in DC fields is given by $\Gamma_{\text {g.s.decay }}=-F \int_{\text {B.Z. }} \frac{d \boldsymbol{k}}{(2 \pi)^{D}} \frac{1}{2 \pi} \ln \left(1-\mathcal{P}_{\boldsymbol{k}}\right)$ where $\boldsymbol{k}$ is the momentum in the Brillouin zone 14. Expanding this with $\mathcal{P}$ to the lowest order gives the total production rate of electron-hole pairs, c.f. Eq. (29).

The time evolution of the doublon density

$$
d(t)=\frac{1}{L} \sum_{i=1}^{L}\left\langle n_{i \uparrow} n_{i \downarrow}\right\rangle(t) .
$$

can be related to the total production rate. As we apply a strong electric field to the ground state, doublon density increases from its ground state value. For a continuously applied electric field, we assume that doublon density increases linearly in time, and its increase within a time interval $\Delta t$ is given by

$$
\Delta d \sim \Delta t \Gamma .
$$

Again, this expression is approximate because (a) we ignore the production of multiple pairs and (b) we assume that the state $|p ; \Phi\rangle$ has precisely one additional doublon compared with the ground state, which is a natural assumption when $U$ is large.

Next, we comment on the validity of our method. It is important to note that although we use an exact result (the Bethe ansatz), the calculated production rate is only approximate. One origin of error lies in the LandauDykhne formula itself. It is only valid when excitations are rare events, i.e., $\mathcal{P}_{p} \ll 1$. This means that we can only use Eq. (24) when field strength is not too large compared with the Schwinger limit $F_{\text {th }}$ and photon frequency is below the resonance frequency $\Omega<\Delta_{\text {Mott }}$. A related issue is that the $F_{0} \sim U$ resonance [24] is ignored in static electric fields.

Another important omission is the effect of quantum interference between multiple tunneling events. This is known as the Stokes phenomenon and has been studied in various time-dependent problems (e.g., Ref. [16, 40, 46]). In the present problem of a driven Mott insulator, this is related to the pair annihilation process of doublon-hole pairs. In Ref. [16], the effect of pair annihilation and the resulting quantum interference was studied via mapping into an effective quantum walk. Quantum interference may lead to several anomalous behaviors. An example is dynamical localization in energy space [16]. The LandauDykhne method ignores the interference effect, and we will see the outcome of this later in Section IVB while presenting a comparison with numerical results.

\section{DIELECTRIC BREAKDOWN IN DC FIELDS (SCHWINGER LIMIT)}

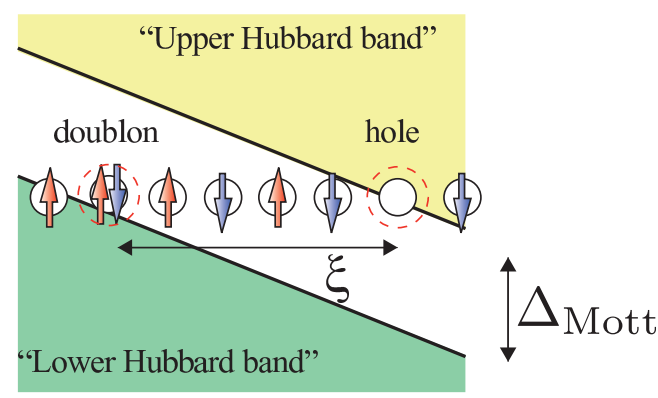

FIG. 4: (color online) Schematic rigid band description of the dielectric breakdown of Mott insulators in DC-electric fields. The upper and lower "Hubbard bands" are tilted with static potential $V(x)=F_{0} x$ and quantum tunneling starts to occur when the energy drop $F_{0} \xi$ between the doublon pairs separated by $\xi$ becomes comparable to the excitation gap $\Delta_{\text {Mott }}$.

The case of DC electric fields was studied in Ref. [15], and the Schwinger limit (=tunneling threshold) was obtained and compared with td-DMRG. In DC fields $F=F_{0}$, when we perform the integrals in Eqs (26) and (27), we notice that the contribution from path $\Gamma_{1}$ vanishes because $F$ is always real. Thus, we have $\operatorname{Im} \mathcal{D}_{p}=\int_{0}^{p_{\mathrm{c}}} \Delta E(i l) d l / F_{0}$ which leads to the threshold form

$$
\mathcal{P}_{p}=\exp \left(-\pi \frac{F_{\mathrm{th}}}{F_{0}}\right)
$$

with

$$
F_{\mathrm{th}}=\frac{2}{\pi} \int_{0}^{p_{\mathrm{c}}=1 / \xi} \Delta E(i l) d l .
$$

This coincides with earlier results [15]. Further physical insight can be gained using an approximation (accurate for small $U$ [50]) such as

$$
\Delta E(i l) \simeq \Delta_{\text {Mott }} \sqrt{1-(\xi l)^{2}},
$$

which makes the integral in Eq. (35) trivial. Then, we obtain the Schwinger limit

$$
F_{\mathrm{th}} \simeq \frac{\Delta_{\mathrm{Mott}}}{2 \xi}
$$


As explained above (section 【), attempt frequency is given by $1 / T=F_{0} / 2 \pi$, and production rate becomes

$$
\Gamma_{p}=\frac{F_{0}}{2 \pi} \exp \left(-\pi \frac{F_{\mathrm{th}}}{F_{0}}\right) \quad \text { (DC-fields). }
$$

We note that the case of a DC field is special in the sense that production rate has no $p$-dependence. This is because all states experience the same tunneling event. A state with momentum $p$ drifts in the momentum space as $p+F_{0} t$ and undergoes tunneling around $p+F_{0} t \sim 0$ when the gap becomes smallest.

The interpretation of the result can be simplified if we employ the rigid band picture (Fig. (4). The rigid band picture simply views a Mott insulator as a band insulator with the role of conduction and valence bands played by the upper and lower "Hubbard bands" with the "band gap" $\Delta_{\text {Mott }}$. To make a pair with energy $\Delta_{\text {Mott }}$, the doublon and hole must be separated from each other in a virtual process until they become real (on-shell). The separation is on the order of $\Delta_{\text {Mott }} / F_{0}$ and the probability for this to happen is given by Green's function, i.e., $\mathcal{P} \propto G\left(\kappa \Delta_{\text {Mott }} / F_{0}\right)$ with $\kappa=\pi / 2$. Because Green's function decays exponentially in the Mott insulating phase [Eq. (11)], we obtain a production rate exponentially dependent on the electric field, i.e., Eq. (38).

If we compare Eq. (37) with Schwinger's threshold in QED [34, 35], we notice that the correlation length $\xi$ plays the role of the Compton wavelength $\lambda=h / m_{e} c$. In the small $U$ limit, correlation length (soliton length) is $\xi=2 v_{\text {eff }} / \Delta_{\text {Mott }}$ with $v_{\text {eff }}=2+U / 2 \pi+\cdots$ the speed of the charge excitations [45]. In this limit, we recover the Landau-Zener result [13]

$$
\Gamma_{p}=\frac{F_{0}}{2 \pi} \exp \left(-\pi \frac{\left(\Delta_{\mathrm{Mott}} / 2\right)^{2}}{v_{\mathrm{eff}} F_{0}}\right) \quad(\mathrm{DC}, \text { Small } U)
$$

analogous to the Schwinger mechanism in QED with $F_{\text {th }} \propto(\text { gap })^{2}$ [34, 35].

In the large $U$-limit, we have $\xi^{-1} \sim \ln (U / g \tau)(g \sim$ 4.3) 45] and production rate shows an interesting power law behavior

$$
\Gamma_{p}=\frac{F_{0}}{2 \pi}\left(\frac{g \tau}{U}\right)^{\frac{\pi}{2} \frac{U}{F_{0}}} \quad(\text { DC, Large } U)
$$

with the hopping parameter $\tau$ recovered. This result can be understood intuitively from a strong-coupling argument. After pair creation in the Mott insulating ground state, the doublon must hop $\Delta_{\text {Mott }} / F_{0} \simeq U / F_{0}$ sites away from the accompanying hole to become on-shell. The amplitude decreases by a factor $\left(\frac{\tau}{U}\right)$ for each hopping, and thus, we are led to Eq. (40). In Fig. 5 we plot the $U$ dependence of the threshold (Schwinger limit).

\section{KELDYSH CROSSOVER IN AC FIELDS}

Next, we study a situation where a strong laser represented by

$$
F(t)=F_{0} \sin \Omega t
$$

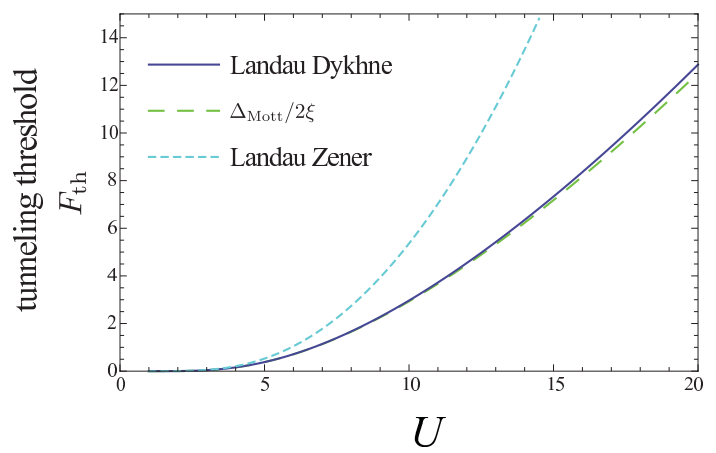

FIG. 5: (color online) Schwinger limit (= tunneling threshold) 15] of the 1D Hubbard model at half-filling. In (c), the solid line is the Landau-Dykhne result given by Eq. (35), whereas the dashed line is its approximate form Eq. (37). The LandauZener result in Eq. (39) with $v_{\text {eff }}=2$ is plotted as a dotted line. The Landau-Zener result is only accurate for a small $U$.

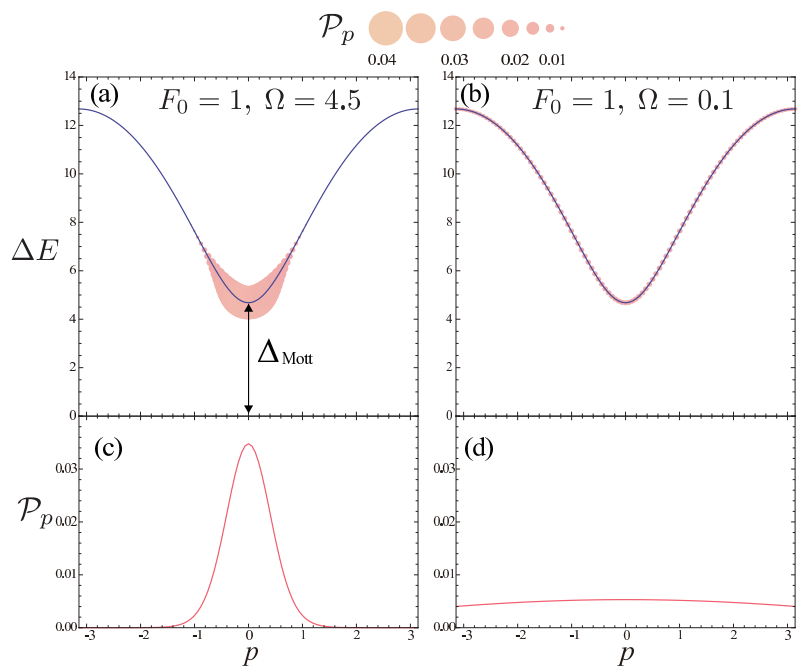

FIG. 6: (color online) Tunneling probability of the dh-pair obtained for the $U=8$ Hubbard model in an AC fields. (a) and (c) correspond to $F_{0}=1.0$ and $\Omega=4.5$, respectively, which are in the multi-photon regime, while (b) and (d) are for $F_{0}=1.0$, and $\Omega=0.1$, respectively, which are in the quantum tunneling regime. In (a) and (b), the tunneling probability is indicated by the size of the circle plotted on top of the dh-pair spectrum.

( $F_{0}$ : field strength, $\Omega$ : photon energy) is applied to a Mott insulator. Experimentally, this models photocarrier injection, which is the initial process in the photo induced insulator to metal phase transition [1, 2]. In standard photocarrier injection, the laser's photon energy $\Omega$ is set to the absorption peak, which is slightly above the Mott gap $\Delta_{\text {Mott }}$. However, herein, we are interested in the nonlinear process induced by subgap lasers, i.e., $\Omega<\Delta_{\text {Mott }}$.

The tunneling probability $\mathcal{P}_{p}$ as well as production rate $\Gamma_{p}$ can be calculated using Eq. (24) and Table [1] We note that the sign in the Jacobian is determined so that $\operatorname{Im} \mathcal{D}_{p 1,2} \geq 0$ is satisfied. 

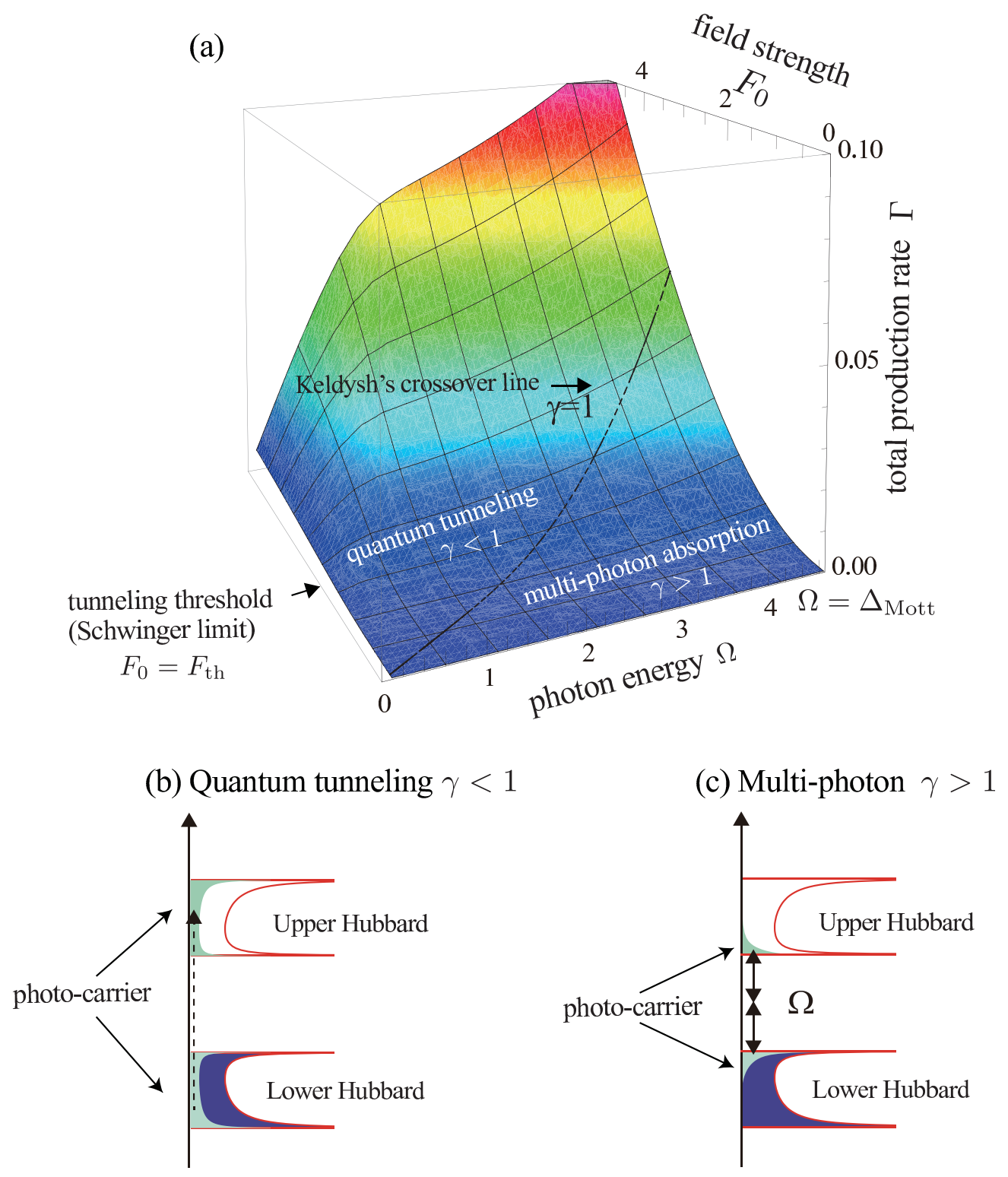

FIG. 7: (color online) (a) Total production rate of the $U=8$ Hubbard model in an AC field. The dashed line corresponds to Keldysh crossover $\gamma=1$, where the excitation mechanism changes from multi-photon absorption to quantum tunneling. Schwinger limit is $F_{\mathrm{th}}=1.668$. (b), (c) Schematic pictures of the nonlinear excitation in the two regimes. In the quantum tunneling regime (Fig. (b)), the doublon-hole distribution becomes momentum independent, which means that the "upper Hubbard band" becomes populated by photocarriers.

An interesting feature of photocarriers generated by nonlinear subgap lasers is that one can control the distribution of doublon-hole pairs by changing the photon en$\operatorname{ergy} \Omega$. In Fig.6. we plot the momentum resolved tunneling probability for the $U=8$ Hubbard model. We notice that the distribution in the momentum space changes drastically when photon energy is changed. When $\Omega$ is large, the generated dh-pair is localized near the gap $\Delta E \sim \Delta_{\text {Mott }}$. The peak becomes broader as the field strength $F_{0}$ becomes larger. On the other hand, when $\Omega$ is small, the dh-pair becomes uniformly distributed in the $p$ space. In the small $\Omega$ limit, we approach the
DC field case, where tunneling probability has no $p$ dependence, c. f., Eq. (38). In fact, the excitation mechanisms in the two regimes are different. For small $\Omega$ and large field strength, quantum tunneling is responsible for dh-pair creation. On the other hand, when $\Omega$ is large, multi-photon absorption is the excitation mechanism. If we change photon energy and laser strength, there is a crossover between the two regimes, which is the Keldysh crossover 31 mentioned in the Introduction. We can directly see this from the analytical expression of the $p=0$ 
tunneling probability

$$
\begin{aligned}
& \mathcal{P}_{p=0} \simeq \exp \left(-\frac{2 \Delta_{\mathrm{Mott}} \gamma}{\Omega} f(\gamma)\right) \quad(\text { AC-fields }), \\
& \rightarrow\left\{\begin{array}{cc}
\left(\frac{F_{0} \xi}{h \Omega}\right)^{2 \frac{\Delta_{\mathrm{Mott}}}{\Omega}} & \gamma \gg 1, \\
\exp \left(-\frac{\pi}{2} \frac{\Delta_{\mathrm{Mott}}}{\xi F_{0}}\left(1-\frac{\pi}{16} \gamma^{2}+\ldots\right)\right) & \gamma \ll 1,
\end{array}\right.
\end{aligned}
$$

This result is obtained with the help of the approximation in Eq. (36) ( $h \sim 1.47$ and see footnote 52] for function $f)$. We note that this expression is identical to the QED result [37] with a redefinition of Keldysh's adiabaticity parameter [31]

$$
\gamma=\frac{\Omega}{\xi F_{0}} .
$$

The crossover is characterized by the Keldysh line defined by $\gamma=1$. In the multi-photon absorption regime [ $\gamma \ll 1$; Fig. [7(c)], tunneling probability has a power law dependence on field strength. The power $2 \Delta_{\text {Mott }} / \Omega$ is twice the number of absorbed photons. As stated above, photocarriers are generated near the excitation gap.

On the other hand, in the quantum tunneling regime [ $\gg$; Fig. [7(b)], tunneling probability shows a threshold behavior with an exponential suppression. In this regime, the photocarriers are distributed almost equally in the momentum space. In Fig. 7 (a), we plot the total production rate as a function of photon energy and field strength. The Keldysh crossover line is indicated by a dashed line. In the quantum tunneling regime, the production rate quickly increases as field strength exceeds tunneling threshold, which is $F_{\text {th }}=1.668$ for the $U=8$ Hubbard model.

When we compare the present result with those of previous studies on quantum tunneling in AC field backgrounds such as atom ionization [31] and nonlinear QED 36 38, we notice that the Keldysh crossover is quite universal and is not limited to the Hubbard model. Previous studies were conducted on non-interacting systems where excitation occurs between single particle gaps, whereas in the present case, the system strongly interacts and the origin of the excitation gap is a manybody effect. The basic idea of the Keldysh crossover survives in many-body systems and expressions such as tunneling threshold (Schwinger limit) [Eq. (37)] and the Keldysh's adiabaticity parameter (Eq. (44)) are valid where the many-body features are renormalized on the correlation length $\xi$. However, if we carefully consider the long time dynamics, differences between non-interacting systems and many-body systems can be observed. This is examined below in the next subsection.

\section{A. Comparison with numerical results}

To examine the applicability of the Landau-Dykhne method, we compare its result with that of td-DMRG (a)
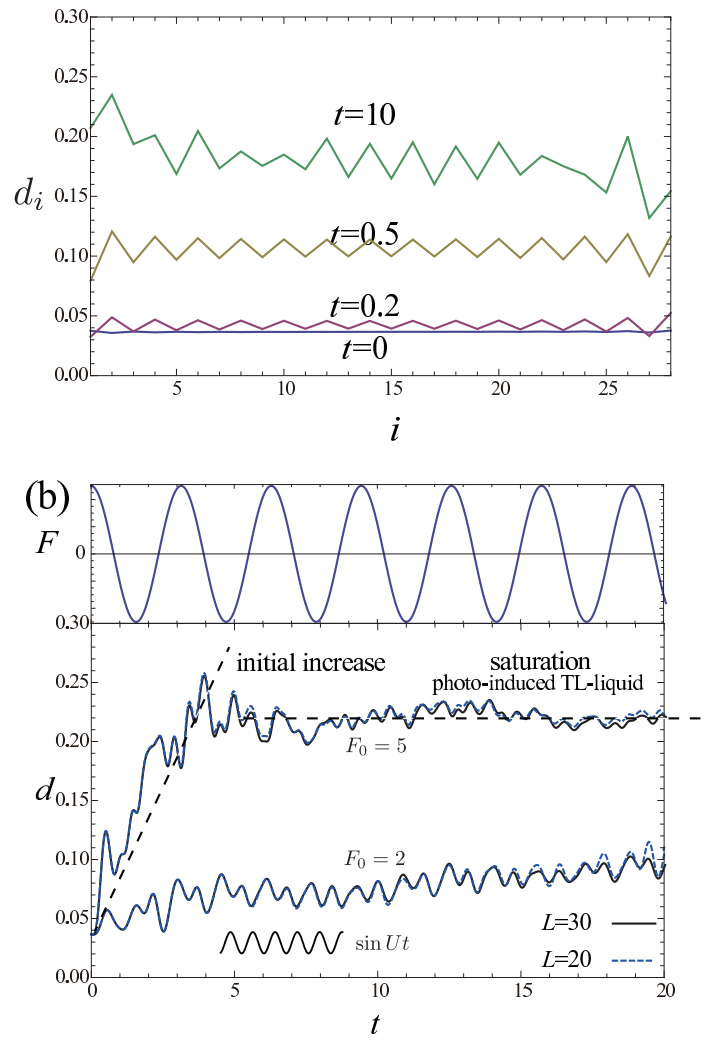

(c)

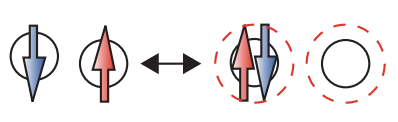

FIG. 8: (color online) (a) Time evolution of doublon density in AC-fields calculated by td-DMRG for the $U=8$ Hubbard model $\left(L=30, F_{0}=4, \Omega=2\right)$. (b) Time evolution of the averaged doublon density $d$. Results for $L=30$ and $L=20$ are plotted. Upper panel is the electric field $F(t)=F_{0} \cos \Omega t$. Doublon density shows intial increase and then saturation occurs. (c) Resonant oscillation between the ground state and the excited state with a neighboring doublon and hole pair.

51]. The 1D Hubbard model on an $L$-site open chain with the Hamiltonian

$$
\begin{aligned}
H(t)= & -\tau \sum_{j, \sigma}\left(c_{j+1 \sigma}^{\dagger} c_{j \sigma}+c_{j \sigma}^{\dagger} c_{j+1 \sigma}\right) \\
& +U \sum_{j} n_{j \uparrow} n_{j \downarrow}+F(t) \sum_{j} j n_{j} .
\end{aligned}
$$

is studied. This Hamiltonian is identical to the previous one [Eq. (5)] in the infinite size limit, and is related through a gauge transformation. We start time evolution from the ground state $|0\rangle$ obtained by the finite-size method and apply the electric field for $t>0$. In the calculation, the DMRG Hilbert space is $m=200$, the time step is 0.01 , and the length of the chain is $L=20,30$.

There are several interesting features in the time evolution of the doublon density

$$
d_{i}=\left\langle n_{i \uparrow} n_{i \downarrow}\right\rangle
$$



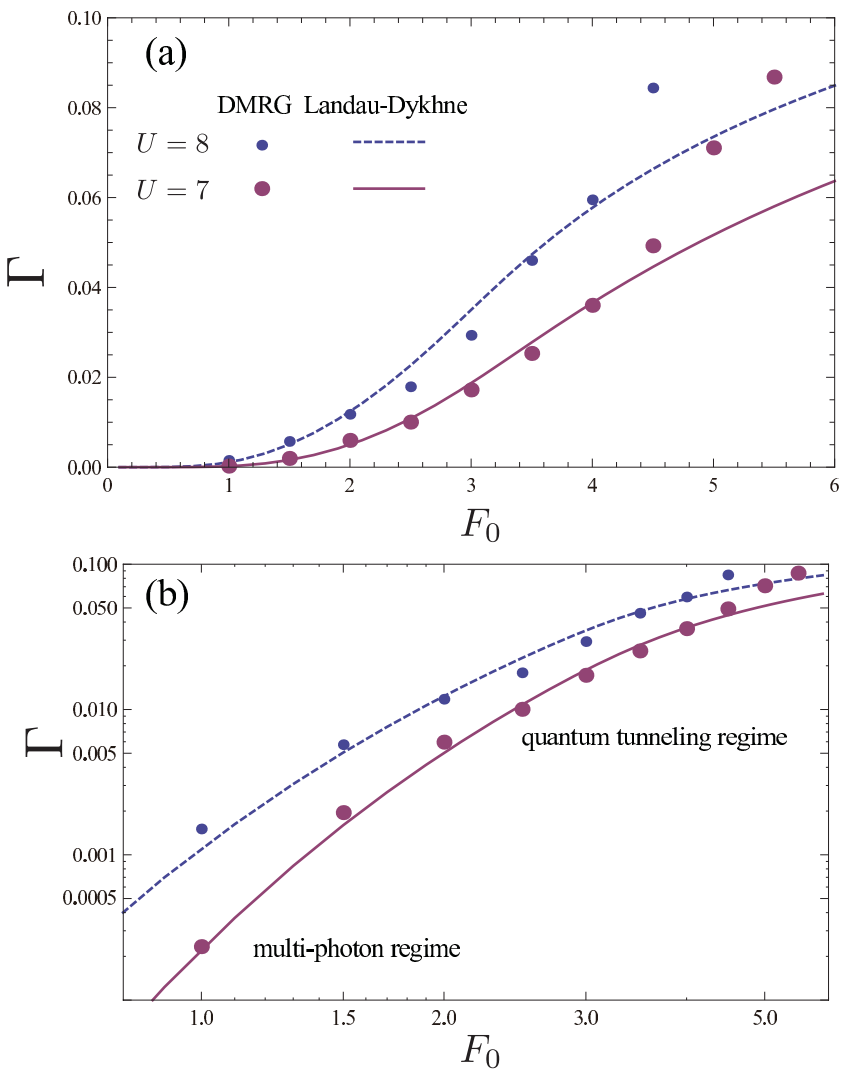

FIG. 9: (color online) Total production rate for the $U=8,7$ Hubbard model with photon energy $\Omega=1$ plotted against field strength $F_{0}((\mathrm{~b})$ is the logarithmic plot). The td-DMRG result is obtained using the fitting of Eq. (48), which is compared with the Landau-Dykhne result. Photon energy is $\Omega=1$ and system size is $L=30$.

as plotted in Fig. 8 First, in addition to the overall increase, a density wave of doublons of the form $d_{i}(t) \sim d(t)+(-1)^{i} \delta d(t)$ occurs. We note that the electron density $n_{i}=\sum_{\sigma}\left\langle n_{i \sigma}\right\rangle$ does not show such modulations. In addition, the finite-size effect is present in the calculation. This shows up in the doublon density as a difference between the edge and bulk values. To eliminate the finite-size effect as well as the density wave from the analysis, we define the averaged doublon density $d(t)$ as an average within the central region of the chain. For example, for an $L=30$ system, the average is taken over the middle 10 sites. The time evolution of the averaged doublon density is plotted in Fig. 8 (b) for $L=20$ and $L=30$, and we see that size dependence is not large after averaging. The averaged doublon density shows a fast oscillation with a period of $2 \pi / U$. One possible explanation for the appearance of the density wave and time oscillation is the locality of dh-pair production. The correlation length $\xi$ gives the typical size of the dhexcitation created by electric fields. Because $\xi$ is short when $U$ is large, e.g., $\xi<1$ when $U>10$ [see Fig. 3(b)], most of the dh-excitation takes place between neighboring sites as in Fig. 8 (c). The energy difference between the ground state this state is $U$, which leads to temporal oscillation.

The averaged doublon density plotted in Fig. 8 (b) shows an increase and the speed of increase becomes larger in stronger fields. After the initial increase, we notice that saturation takes place although the AC field is still present. If the field strength is sufficiently large, the long-term driven state is a metallic state called the "photo-induced Tomonaga-Luttinger-like liquid" 22]. In this state, it was numerically shown by calculating correlation functions that spin-charge separation takes place. However, in this study, we restrict ourselves to nonlinear dh-pair creation, which is responsible for the initial increase in doublon density. The production rate of the dh-pairs is obtained from numerical data by employing the fitting

$$
d(t)=d_{0}+a(\tanh (b t)+1)
$$

and production rate is identified as the initial slope

$$
\Gamma_{\mathrm{DMRG}}=a b .
$$

In Fig 9 we plot the total production rate obtained by td-DMRG and compare it with the analytical result calculated from the Landau-Dykhne formula, i.e., Eq. (29). Although the fitting is difficult owing to the fast oscillation of doublon density, the results seem to agree quite well. The numerical result agrees with the LandauDykhne result in the weak field regime up to $F_{0}<4$. The deviation at large $F_{0}$ is expected because the LandauDykhne formula is known to work only near the adiabatic limit, i.e., $\mathcal{P} \ll 1$. In the log-log plot, we clearly see the crossover from the weak field multi-photon absorption regime, with a power law behavior, to the quantum tunneling regime.

\section{B. Single pulse}

Using the Landau-Dykhne method, we can extend the analysis to the case of a single pulse. There are two motivations. First, recent ultra fast pulse lasers are becoming so short that a single pulse excitation is now experimentally realizable. Second, we wish to further examine the validity of the Landau-Dykhne method from a theoretical perspective. In this regard, a single pulse is ideal because problems such as saturation and driven steady states do not occur, as is the case with a continuous AC field. The pulse we study here is

$$
F=F_{0} \cosh ^{-2}(t / \sigma)
$$

and the Jacobian needed for the calculation is given in Table \. We set the duration to $\sigma=2$.

In Fig. 10(b), we plot the time evolution of doublon density for a $1 \mathrm{D} U=10$ Hubbard model on an open $L=50$ chain calculated by td-DMRG. Doublon density initially increases and then oscillates with a period 

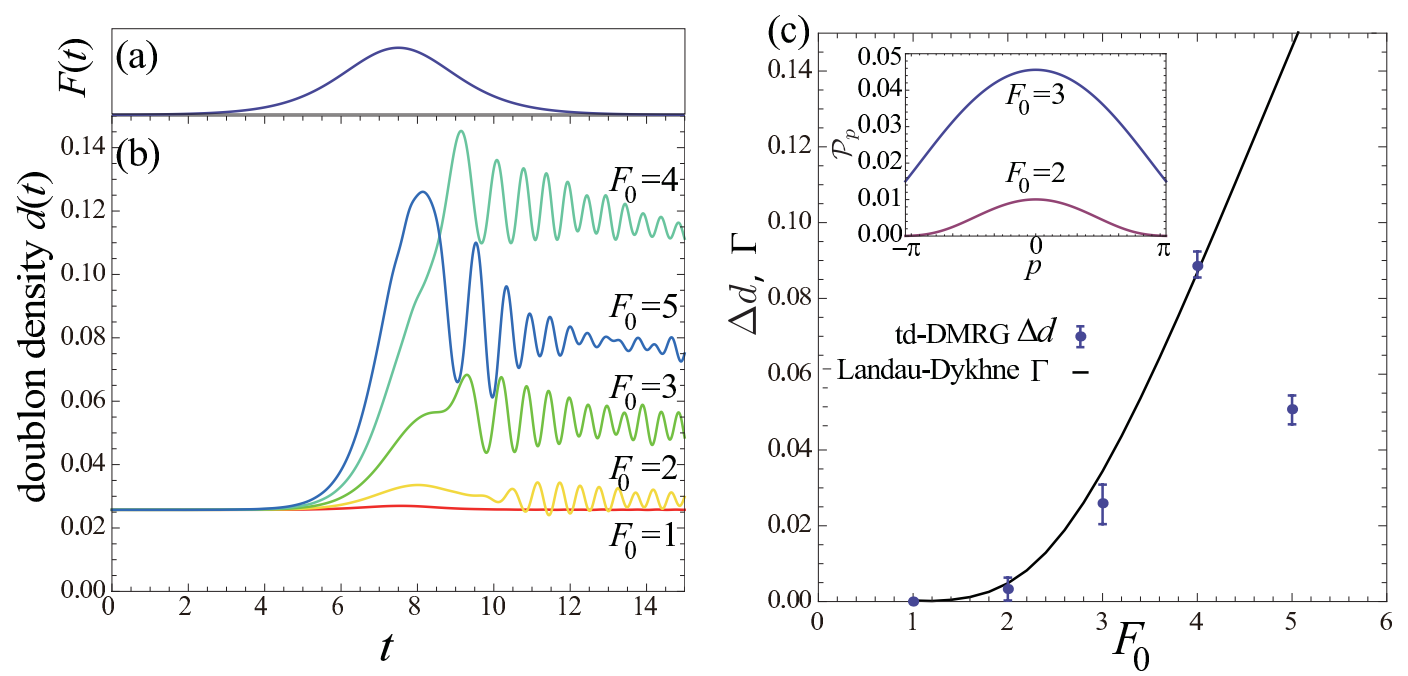

FIG. 10: (color online) (a) Shape of pulse field with duration $\sigma=2$. (b) Time evolution of the doublon density $d(t)$ for the $U=10$ Hubbard model obtained by td-DMRG. (c) Increase in the doublon density $\Delta d$ obtained by td-DMRG and the total production $\Gamma$ obtained by the Landau-Dykhne method. See main text for the error bar. Inset: $p$-resolved distribution function of doublon-hole pairs obtained by the Landau-Dykhne method.

roughly given by $T=2 \pi / U$. This oscillation is similar to the AC field case seen in Fig. 8(b). Increase in the doublon density $\Delta d$ is plotted in Fig. 10(c), where the error bars are determined by the oscillation width near $t=15$. At $F_{0}<4, \Delta d$ shows a monotonic increase, whereas it shows an irregular decrease at $F_{0}=5$.

Let us compare the numerical result with LandauDykhne results. In the inset of Fig. 10(c), the momentum resolved tunneling probability $\mathcal{P}_{p}$ is plotted. The distribution has a peak at $p=0$ and broadens as the field becomes stronger. Eventually, as seen in the $F_{0}=3$ data, high-energy dh-pairs with momentum $p \sim \pm \pi$ become excited as well. We compare the total production rate $\Gamma$ obtained from the Landau-Dykhne approach with $\Delta d$. Comparison with td-DMRG revealthat the LandauDykhne method gives the correct threshold behavior and is reliable even for relatively strong electric fields. However, the Landau-Dykhne method does not capture the irregular drop at $F_{0}=5$ and we detect a small deviation at $F_{0}=3$. As mentioned above, the Landau-Dykhne method ignores the interference effect due to multiple quantum tunneling [46], which becomes important as pair annihilation of doublons and holes become activated. In fact, when the effect of interference is strong, quantum tunneling can be suppressed by dynamical localization in the energy space 23]. Such an effect is expected to become important when the field is strong, and we think that it explains the differences between the numerical and Landau-Dykhne approach results.

\section{EXPERIMENTAL FEASIBILITY}

In this section, we discuss the experimental feasibility of the nonlinear doublon excitations predicted in this study. Candidates of physical systems range from fermionic cold atoms to solid state crystals. For example, if we use fermionic cold atoms in an optical lattice, the present theory can be experimentally verified by inducing a time-dependent tilt of the lattice. This mimics the effect of an electric field and a direct measurement of the doublon density is possible as well [10, 11]. In solid states, we must estimate the threshold field strengths of candidate materials and compare them with the peak field strengths of present day lasers. In addition, because doublon density is not a directly observable quantity in solids, we must seek alternative detection methods. We discuss these issues below.

\section{A. Threshold of 1D Mott insulators}

Pump-probe experiments with a terahertz $(\mathrm{THz})$ laser are ideal setups to verify the Keldysh crossover described in section IV] Current THz pulse lasers can be as strong as $1 \mathrm{MV} / \mathrm{cm}[6,7]$ and the typical photon energy is $\Omega=$ $4 \mathrm{meV}$ corresponding to $1 \mathrm{THz}$.

On the material side, the candidate 1D Mott insulator ranges from organic crystals to cuprates. The tunneling thresholds of several materials estimated by the LandauDykhne + Bethe ansatz method are shown in table II. In the list, the material with the smallest threshold is ET- $\mathrm{F}_{2}$ TCNQ (ET-salt). Let us discuss the possibility of generating dh-pairs in this material with a $\mathrm{THz}$ laser. The tunneling threshold (Schwinger limit) for the material is $E_{\mathrm{th}}=3 \mathrm{MV} / \mathrm{cm}$. When using a laser with peak strength $E_{\text {laser }}=1 \mathrm{MV} / \mathrm{cm}[\underline{6}, 7]$, we are still below the 


\begin{tabular}{|c|ccc|ccc|}
\hline & $\tau(\mathrm{eV})$ & $U(\mathrm{eV})$ & $a(\ddot{A})$ & $\Delta_{\text {Mott }}(\mathrm{eV})$ & $\xi(a)$ & $E_{\mathrm{th}}(\mathrm{MV} / \mathrm{cm})$ \\
\hline $\mathrm{ET}-\mathrm{F}_{2} \mathrm{TCNQ}$ & 0.1 & 1 & 10 & 0.7 & 1.1 & 3 \\
{$\left[\mathrm{Ni}(\mathrm{cnxn})_{2} \mathrm{Br}_{\mathrm{Br}}\right]$} & 0.22 & 2.4 & 5 & 1.6 & 1.0 & 16 \\
$\mathrm{Sr}_{2} \mathrm{CuO}_{3}$ & 0.52 & 3.1 & 4 & 1.5 & 2.1 & 9 \\
\hline
\end{tabular}

TABLE II: Mott gap $\Delta_{\text {Mott }}$, correlation length $\xi$, and tunneling threshold (Schwinger limit) $F_{\text {th }}=e a E_{\text {th }}$ calculated by the Landau-Dykhne method with the Bethe ansatz. Material parameters ( $\tau$ : hopping, $U$ : onsite repulsion, $a$ : lattice constant) are obtained from Ref. [2] (ET-F $\left.\mathrm{F}_{2} \mathrm{TCN}\right)$ and Ref. [53] ([Ni(cnxn) $\left.\left.)_{2} \mathrm{Br}\right] \mathrm{Br}_{2}, \mathrm{Sr}_{2} \mathrm{CuO}_{3}\right)$.

threshold, and the tunneling probability is

$$
\begin{aligned}
\mathcal{P} & =\exp \left(-\pi \frac{E_{\text {th }}}{E_{\text {laser }}}\right) \\
& \sim 8 \times 10^{-5} \quad \text { (for ET-salt). }
\end{aligned}
$$

This is too small to trigger photo-induced metallization, and therefore, a stronger light source is needed. Quite recently, amplification of laser field strength using a metamaterial structure has been proposed [8, 55]. It is reported that peak strength can be as large as $E_{\text {metamaterial }}=4 \mathrm{MV} / \mathrm{cm}[8]$. If this technique can be applied, tunneling probability becomes

$$
\begin{aligned}
\mathcal{P} & =\exp \left(-\pi \frac{E_{\mathrm{th}}}{E_{\text {metamaterial }}}\right) \\
& \sim 0.1 \quad \text { (for ET-salt). }
\end{aligned}
$$

This means than one can perform a $~ 10 \%$ photodoping with a single pulse. This value is large enough that one can trigger a photo-induced phase transition.

\section{B. Angle resolved photoemission spectroscopy of the upper Hubbard band in the quantum tunneling regime}

As stated in Section IV [see Fig. 7(b), (c)], the distribution of the produced dh-pairs changes drastically when the laser is shifted from the multi-photon absorption regime to the quantum tunneling regime. In the quantum tunneling regime, high energy dh-pairs are generated. This means that the entire "upper Hubbard band" becomes populated by photocarriers. Thus, using a strong $\mathrm{THz}$ laser as the pump, it is possible to study the band structure of the upper Hubbard band with a real time angle resolved photoemission spectroscopy technique. A necessary condition is that the Keldysh parameter (Eq. (44))

$$
\gamma=\frac{\Omega}{\xi F_{0}}
$$

is well below unity. From Table【 the Keldysh crossover field strength for a $1 \mathrm{THz}$ laser $(\Omega=4 \mathrm{meV})$ is

$$
\begin{aligned}
E_{\text {crossover }} & =\frac{\Omega}{a \xi} \\
& \sim 4 \times 10^{-2} \mathrm{MV} / \mathrm{cm} \quad \text { (for ET-salt) }
\end{aligned}
$$

and the pump laser must exceed this strength. With stronger fields, more photocarriers are excited and the measurement is expected to be more feasible.

\section{Dielectric breakdown and nonlinear transport}

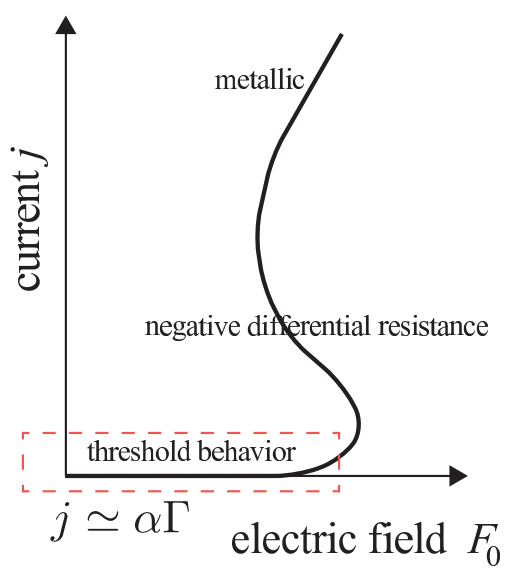

FIG. 11: (color online) Typical $I V$-characteristics of strongly correlated insulators. We have a threshold behavior, negative differential resistance and a transition to a metallic state. The present theory is only applicable to explain the threshold behavior in the small current regime shown in the dashed box.

In nonlinear transport experiments, a threshold behavior in the $I V$-characteristics is found in many correlated insulators. Materials range from Mott insulators [3], charge-ordered systems [4], and materials showing a neutral-ionic transition [5]. In Fig 11, we plot typical $I V$-characteristics. In many cases, a threshold behavior as well as a region with negative differential resistance is present. Let us make a comment on the threshold behavior appearing in the small current regime, i.e., dielectric breakdown [region inside the dashed line in Fig 11].

Although doublon density is not a measurable quantity, we can relate the dh-pair production rate to current itself. Theoretical studies [17, 19] suggests that, in nonlinear transport of Mott insulators, current has two major contributions

$$
J=\sigma F_{0}+\alpha \Gamma .
$$

The first term is the standard linear response due to thermal carriers with a temperature dependence $\sigma_{\text {thermal }} \propto$ 
$e^{-\Delta_{\text {Mott }} / T}$. The second term is proportional to the dhpair production rate with a nonperturbative threshold form

$$
\Gamma=\frac{1}{2 \pi} \exp \left(-\pi \frac{F_{\mathrm{th}}}{F_{0}}\right) F_{0} .
$$

$\alpha$ is a nonuniversal proportionality constant which depends on the coupling to the electrode and other factors.

One may think that photocarriers induced by dh-pair production may contribute to linear response, i.e., the term $\sigma F_{0}$. This is not true in dielectric breakdown occuring in the quantum tunneling regime. The reason is because the dh-pairs are in an "infinite temperature state" 56]. As shown in Section III. the distribution of dh-pairs has no momentum-, and thus energy-, dependences. This corresponds to an infinite temperature state, i.e., $e^{-E / k_{B} T}$ with $T \rightarrow \infty$. The conductivity of an infinite temperature state is zero, and therefore, there are no linear reponse contribution in the quantum tunneling regime. Instead, the current is dominated by the second term in Eq. (54) which is proportional to the dhpair production rate. The doublons and holes are pair created, separated from each other by the electric field, and are measured as current when they are absorbed by electrodes. This feature is consistent with numerical results obtained in a static system coupled to electrodes [19].

After dielectric breakdown, the $I V$-characteristics show interesting nonlinear behaviors. Although this is far beyond the applicability of the present theory, let us consider existing literatures. If voltage drop is measured as a function of current, there is a regime where negative differential resistance is realized [3 [5]. The origin of negative differential resistance is not fully understood yet. It was pointed out, with a careful comparison with experimental data, that the temperature increase of the sample due to Joule heating can explain it [4]. They suggest that negative differential resistance occurs when the temperature dependence of conductivity is large. A related theoretical paper explained negative differential resistance in disordered films via the heating mechanism [54]. A more dramatic proposal is based on nonequilibrium first-order phase transition proposed by Ajisaka et. al. 25], where negative differential resistance is explained through a phase bi-stability. Negative differential resistance was also found in a model in high energy physics, namely the supersymmetric QCD in the large $N$ limit [26]. A microscopic understanding of the nonlinear transport properties of correlated systems from a universal viewpoint is an interesting callenge.

\section{Optical sum rule}

From Eq. (54), we can derive an interesting relationship between the optical sum rule and the doublon production rate in DC-electric fields. Here, we consider the low temperature case where contributions from thermal carriers are negligible, and the field strength is below the threshold [region inside dashed line in Figure 11.

The optical sum rule is commonly used by experimentalists as a means to "measure" carrier density from the absorption spectrum[1]. The optical sum rule for the Hubbard model (e.g., [49]) states that the frequency integral of the absorption spectrum, which we call $N_{\text {eff }}$ following Ref. [1], is related to kinetic energy as follows:

$$
N_{\mathrm{eff}} \equiv \int_{0}^{\infty} \frac{d \omega}{\pi} \sigma_{1}(\omega)=-\frac{1}{2 L} \bar{K}
$$

Here, $\bar{K}$ is the time average of the expectation value of the kinetic term in the Hamiltonian, i.e.,

$$
K(t)=\left\langle\Psi(t)\left|-\tau \sum_{j, \sigma}\left(e^{i \Phi} c_{j+1 \sigma}^{\dagger} c_{j \sigma}+e^{-i \Phi} c_{j \sigma}^{\dagger} c_{j+1 \sigma}\right)\right| \Psi(t)\right\rangle .
$$

Below the threshold, dh-pair creation is a rare event and current is very small. In this regime, energy dissipation by external degrees of freedom, e.g., phonons, is negligible and we can use Joule's relation

$$
\frac{d}{d t}\langle H(t)\rangle=L J F_{0}
$$

to relate change in energy

$$
\langle H(t)\rangle=K(t)+U L d(t)
$$

to the current $J$. Using Eq. (54) as well as $\frac{d}{d t} d(t)=\Gamma$, we obtain

$$
\frac{d}{d t} N_{\text {eff }}=\left(U-\alpha F_{0}\right) \Gamma
$$

This formula states that, in static electric fields, optical sum $N_{\text {eff }}$ increases linearly in time and the speed is proportional to the dh-pair production rate. This relationship opens a way to measure doubon increase by optical experiments.

\section{CONCLUSION}

We developed an analytical theory for nonlinear pair excitations of doublons and holes in a 1D Mott insulator subject to DC, AC and pulse electric fields. The theory is based on the Landau-Dykhne method combined with the Bethe ansatz. In an AC-field, the theory predicts a crossover between multi-photon absorption and quantum tunneling when the strength and photon energy of the field changes. Comparison with numerical results by tdDMRG shows that the analytical theory is reliable up to moderate field strength.

There are several limitations in our theory. Perhaps one of the most important open issues is the treatment of temperature effects. It is unclear if the tunneling probability has a direct temperature dependence. Numerical results obtained by nonequilibrium DMFT suggest 
no or very small temperature dependence in the quantum tunneling regime [17], whereas strong temperature dependence of the threshold is seen in a dielectric breakdown experiment [3]. Impact ionization, an avalanche like cascade growth of carriers due to field induced acceleration, may be important in understanding these experiments. The origin of negative differential resistance and the properties of the nonequilibrium steady state is another important open problem (Section $\mathrm{VC}$ ). We think that trying to answer these problems will lead to important innovations in nonequilibrium manybody physics.

We acknowledge Philipp Werner, Kunio Ishida, David Pekker, Rajdeep Sensarma, Li Gao, Stuart Parkin, Gerald Dunne and Eugene Demler for valuable discussions. TO acknowledges support from Grant-in-Aid for Young Scientists (B), CUA and ITAMP.
Note added: After the submission of the initial version of the manuscript, Lenarčič and Prelovšek published an interesting paper 57. They studied the dielectric breakdown in a spin polarized Mott insulator and found that the threshold has a $F_{\text {th }} \propto \Delta^{3 / 2}$ dependence, which is different from the conventional Landau-Zener form. They pointed out that the origin of this difference comes from excitation dispersion. In the half-filled case, the dispersion is relativistic $\omega_{k} \propto\left(k^{2}+\kappa^{2}\right)^{1 / 2}$, i.e., Eq. (36), whereas it is parabolic $\omega_{k} \propto k^{2}+\kappa^{2}$ in the spin polarized model. Using Eq. (35), we can recover the $F_{\text {th }} \propto \Delta^{3 / 2}$ behavior in the spin polarized case. In another article, a variant of Eq. (35) was used to study excitations in the attractive Hubbard model 58. These examples show the wide applicability of the Landau-Dykhne approach in many-body problems.
[1] S. Iwai, M. Ono, A. Maeda, H. Matsuzaki, H. Kishida, H. Okamoto, and Y. Tokura, Phys. Rev. Lett. 91, 057401 (2003).

[2] S. Wall D. Brida, S. R. Clark, H. P. Ehrke, D. Jaksch, A. Ardavan, S. Bonora, H. Uemura, Y. Takahashi, T. Hasegawa, H. Okamoto, G. Cerullo, and A. Cavalleri , Nat. Phys. 7, 114 (2011).

[3] Y. Taguchi, T. Matsumoto, and Y. Tokura, Phys. Rev. B 62, 7015 (2000).

[4] T. Mori, T. Ozawa, Y. Bando, T. Kawamoto, S. Niizeki, H. Mori, and I. Terasaki, Phys. Rev. B 79, 115108 (2009), T. S. Inada, I. Terasaki, H. Mori, and T. Mori, Phys. Rev. B 79, 165102 (2009).

[5] Y. Tokura, H. Okamoto, T. Koda, T. Mitani, and G. Saito, Phys. Rev. B 38, 2215 (1988).

[6] S. Watanabe, N. Minami, and R. Shimano, Opt. Express 19, 1528 (2011).

[7] H. Hirori, A. Doi, F. Blanchard, and K. Tanaka, Appl. Phys. Lett. 98091106 (2011).

[8] Mengkun Liu, Harold Y. Hwang, Hu Tao, Andrew C. Strikwerda1, Kebin Fan, George R. Keiser, Aaron J. Sternbach, Kevin G. West, Salinporn Kittiwatanakul, Jiwei Lu, Stuart A.Wolf, Fiorenzo G. Omenetto, Xin Zhang, Keith A. Nelson, and Richard D. Averitt, Nature 487, 345 (2012).

[9] M. Greiner O. Mandel T. Esslinger T. W. Hänsch, and I. Bloch , Nature 415, 39 (2002).

[10] N. Strohmaier, D. Greif, R. Jördens, L. Tarruell, H. Moritz, T. Esslinger, R. Sensarma, D. Pekker, E. Altman, and E. Demler, Phys. Rev. Lett. 104, 080401 (2010).

[11] D. Greif, L. Tarruell, T. Uehlinger, R. Jördens, and T. Esslinger Phys. Rev. Lett. 106, 145302 (2011).

[12] C. Kollath, A. Iucci, T. Giamarchi, W. Hofstetter, and U. Schollwöck, Phys. Rev. Lett. 97, 050402 (2006).

[13] T. Oka, R. Arita, and H. Aoki, Phys. Rev. Lett. 91, 066406 (2003).

[14] T. Oka and H. Aoki, Phys. Rev. Lett. 95, 137601 (2005).

[15] T. Oka and H. Aoki, Phys. Rev. B 81, 033103 (2010).

[16] T. Oka, N. Konno, R. Arita, and H. Aoki, Phys. Rev. Lett. 94100602 (2005).

[17] M. Eckstein, T. Oka, and P. Werner, Phys. Rev. Lett. 105, 146404 (2010).
[18] M. Eckstein and P. Werner, Phys. Rev. B 84, 035122 (2011).

[19] Y. Tanaka, and K. Yonemitsu, Phys. Rev. B 83, 085113 (2011).

[20] F. Heidrich-Meisner, I. González, K. A. Al-Hassanieh, A. E. Feiguin, M. J. Rozenberg, and E. Dagotto, Phy. Rev. B 82, 205110 (2010).

[21] A. Takahashi, H. Itoh and M. Aihara, Phys. Rev. B 77, 205105 (2008).

[22] T. Oka and H. Aoki, Phys. Rev. B 78, 241104 (R) (2008).

[23] T. Oka, N. Konno, R. Arita, and H. Aoki, Phys. Rev. Lett. 94, 100602 (2005).

[24] S. Sachdev, K. Sengupta, and S. M. Girvin, Phys. Rev. B 66, 075128 (2002).

[25] S. Ajisaka, H. Nishimura, S. Tasaki, and I. Terasaki, Prog. Theor. Phys. 1211289 (2009).

[26] S. Nakamura, Prog. Theor. Phys. 124, 1105 (2010).

[27] M. Imada, A. Fujimori, and Y. Tokura, Rev. Mod. Phys. 70, 1039 (1998).

[28] A. M. Dykhne, Sov. Phys. JETP 14, 941 (1962).

[29] L. Landau and E. Lifshitz, Quantum Mechanics, Vol. 3 (Butterworth-Heinemann, 1981).

[30] J. P. Davis and P. Pechukas, J. Chem. Phys. 64, 3129 (1976).

[31] L. Keldysh, JETP 20, 1307 (1965).

[32] N.B. Delone, and V.P. Krainov, Multiphoton Processes in Atoms, (Springer, 2000).

[33] M. Wilkinson and M. A. Morgan, Phys. Rev. A 61, 062104 (2000).

[34] W. Heisenberg and H. Euler, Z.Physik 98, 714 (1936).

[35] J. Schwinger, Phys. Rev. 82, 664 (1951).

[36] V. S. Popov, Sov. J. Nucl. Phys. 19, 584 (1974).

[37] V. S. Popov, JETP 34, 709 (1972).

[38] E. Brezin and C. Itzykson, Phys. Rev. D 2, 1191 (1970).

[39] V. S. Popov, Physcs-Uspekhi, 47855 (2004).

[40] C. K. Dumlu and G. V. Dunne, Phys. Rev. Lett. 104 250402 (2010).

[41] G. V. Dunne, "New Strong-Field QED Effects at ELI: Nonperturbative Vacuum Pair Production", arXiv:0812.3163 2 , "Heisenberg-Euler Effective Lagrangians : Basics and Extensions", arXiv:hep-th/0406216. 
[42] T. Oka and H. Aoki, "Nonequilibrium Quantum Breakdown in a Strongly Correlated Electron System" in " Quantum and Semi-classical Percolation and Breakdown in Disordered Solids" edited by A.K. Sen, K.K. Bardhan, B.K. Chakrabarti, (Lecture Note in Physics Vol. 762, Springer-Verlag), (2008) arXiv:0803.0422v1.

[43] T. Oka, "Strong field physics in condensed matter", in proceedings of International Conference on Physics in Intense Fields (PIF 2010), arXiv:1102.2482 1.

[44] F. H. L. Essler, H. Frahm, F. Göhmann, A. Klümper and V. E. Korepin, The One-Dimensional Hubbard Model (Cambridge, 2005).

[45] C. A. Stafford and A. J. Millis, Phys. Rev. B 48, 1409 (1993).

[46] G. S. Vasilev and N. V. Vitanov, Phys. Rev. A 70, 053407 (2004).

[47] T. Fukui and N. Kawakami, Phys. Rev. B 58, 16051 (1998).

[48] Y. Nakamura and N. Hatano, J. Phys. Soc. Jpn. 75, 104001 (2006).
[49] E. Jeckelmann, F. Gebhard, and F. H. L. Essler, Phys. Rev. Lett. 853910 (2000).

[50] Numerically, we find that this holds for large $U$ as well. For $U \sim 20$ the difference is at most $5 \%$.

[51] U. Schollwöck, Rev. Mod. Phys. 77, 259 (2005).

[52] $f(\gamma)=(\mathrm{K}(v)-\mathrm{E}(v)) /(\gamma v), v=\gamma / \sqrt{1+\gamma^{2}}$ where $\mathrm{K}$ and $\mathrm{E}$ are the complete elliptic integral.

[53] N. Tomita and K. Nasu, Phys. Rev. B 63, 085107 (2001).

[54] B. L. Altshuler, V. E. Kravtsov, I. V. Lerner, and I. L. Aleiner, Phys. Rev. Lett. 102, 176803 (2009).

[55] K. Fan, H. Hwang, M. Miu, A. Strikwerda, J. Zhang, A. Sternbach, X. Zhang, K. Nelson, and R. Averitt, APS March meeting (2012) http://meetings.aps.org/link/BAPS.2012.MAR.Q17.11.

[56] P. Werner, private communication.

[57] Z. Lenarčič, and P. Prelovšek, Phys. Rev. Lett. 108, 196401 (2012).

[58] S. Uchino, and N. Kawakami, Phys. Rev. A 85013610 (2012). 